\title{
FLORA VASCULAR Y VIVIENDA DE INTERÉS SOCIAL EN VILLAVICENCIO: CONFLICTOS CON LA CONSERVACIÓN DE LA BIODIVERSIDAD
}

\author{
VASCULAR FLORA AND SOCIAL INTEREST \\ URBANIZATION IN VILLAVICENCIO: CONFLICTS WITH \\ THE BIODIVERSITY CONSERVATION
}

${ }^{1}$ Castro-Arguello G.S.
${ }^{2}$ Cruz-Fernández J.
${ }^{3}$ Ortiz-Moreno M.L.

${ }^{1}$ Estudiante de Ingeniería Agronómica, Universidad de los Llanos, Villavicencio, Colombia

2 Bióloga, estudiante de maestría en Desarrollo Sostenible y Medio Ambiente, Universidad de Manizales, Manizales, Colombia.

${ }^{3}$ Bióloga, MSc en microbiología, doctora en ecología y recursos naturales, Universidad de los Llanos, Villavicencio, Colombia

${ }^{1}$ giovanny.castro@unillanos.edu.co

${ }^{2}$ johanna.cruz@unillanos.edu.co

${ }^{3}$ mlortiz@unillanos.edu.co

\section{RESUMEN}

Colombia presenta alrededor de 24.530 especies de plantas vasculares. Sin embargo, esta riqueza florística está disminuyendo o presentando algún grado de amenaza, debido a que su hábitat se ha reducido por el desarrollo de actividades antrópicas como es la urbanización, minería, agricultura, entre otras. Esta misma tendencia se observa en Villavicencio. Por ello, la presente investigación tiene como objetivo describir la diversidad florística remanente en las rondas hídricas del Caño Zuria y humedal La Madrid ubicadas en el barrio homónimo. Para llevar a cabo la investigación, se realizaron muestreos oportunistas donde se colecto material vegetal en estado reproductivo y se identificaron a especie. Como resultados se encontró que la flora asociada al humedal La Madrid posee 19 especies de plantas vasculares agrupadas en 13 familias, siendo Poaceae la mejor representada. De las especies registradas hay dos que están en la categoría de amenaza preocupación menor (LC), las cuales son Andropogon bicornis y Solanum jamaicense. En la flora asociada a la ronda hídrica del caño Zuria se encontraron 21 especies de plantas vasculares agrupadas en 11 familias, de estas la dominante fue Melastomaceae con seis especies, seguida por Araceae con tres especies. De las especies registradas, Psychotria poeppigiana se encuentra en la categoría 
de amenaza preocupación menor (LC). Los análisis realizados muestran que el desarrollo urbano no sostenible amenaza la biodiversidad de la flora y la oferta de servicios ecosistémicos asociados a las rondas hídricas del caño Zuria y humedal La Madrid en el municipio de Villavicencio.

Palabras Claves: Ronda hídrica, diversidad florística, Villavicencio, Humedal, Caño Zuria.

\section{SUMMARY}

Colombia presents around 24,530 species of vascular plants. However, this floristic richness is declining or showing some degree of threat, because their habitat has been reduced by the development of anthropic activities as urbanization, mining, agrarian and other. This same trend is observed in Villavicencio. For this reason, the present research has as objective to describe the floristic diversity remaining in the water rounds of Caño Zuria and La Madrid wetland located in the neighborhood of the same name. To carry out the research, opportunistic samplings were made where plant material in reproductive state was collected and species were identified. As results, it was found that the flora associated with the La Madrid wetland has 19 species of vascular plants grouped into 13 families, with Poaceae being the best represented. Of the registered species there are two that are in the category of least concern concern (LC), which are Andropogon bicornis and Jamaican Solanum. In the flora associated with the water round of the Zuria canal, 21 vascular plant species were found grouped into 11 families, of which the dominant one was Melastomaceae with six species, followed by Araceae with three species. Of the registered species, Psychotria poeppigiana is in the category of minor concern threat (LC). The analyzes carried out show that unsustainable urban development threatens the biodiversity of the flora and the supply of ecosystem services associated with the water rounds of the Zuria canal and La Madrid wetland in the municipality of Villavicencio.

Keywords: Water round, floristic diversity, ViIlavicencio, Wetland, Zuria river.

\section{Resumen gráfico}

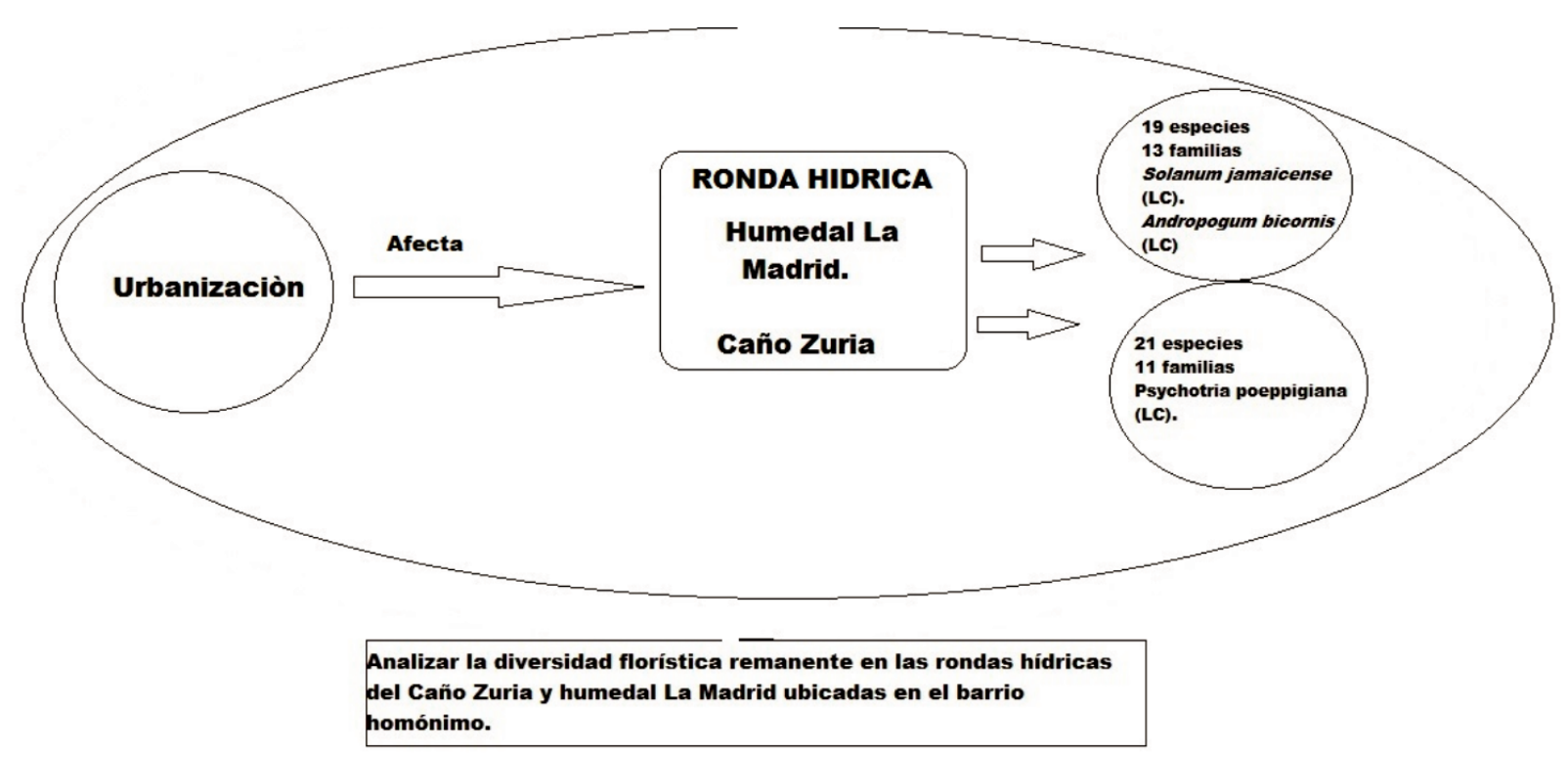

Fuente: autores 


\section{INTRODUCCIÓN}

Colombia presenta una alta diversidad de especies de las que se conocen alrededor de 24.530 plantas vasculares (Bernal et al, 2016). Encontrándose la mayor diversidad en los Andes (Sierra-Giraldo et al, 2013; Uvidia et al, 2015), la cual se considera una de las regiones más diversas del planeta debido a su ubicación geográfica, orografía y variaciones microclimáticas que promueven la generación de microrregiones biogeográficas con alta biodiversidad (Giraldo-Cañas 2001; Myers et al, 2002). Para la Orinoquia colombiana Minorta \& Rangel-Ch. (2014) y Rangel-Ch. (2015) reportan 4.246 especies de plantas vasculares y el Catalogo de plantas y líquenes de Colombia (Bernal et al, 2016) reporta 4.288 especies para el departamento del Meta.

Sin embargo, esta riqueza florística está disminuyendo o presentando algún grado de amenaza, ya que su hábitat se ha reducido debido al desarrollo de actividades como la tala y quema de bosques para ganadería extensiva e intensiva, ampliación de la frontera agrícola, al igual que la urbanización asociada al crecimiento poblacional y la minería (Ortiz-Moreno, 2015; Cruz-Fernández et al, 2017; Pastrana, 2017). Por otra parte, el tráfico ilícito de fauna y flora y la introducción de especies exóticas son amenazas reales a la biodiversidad que son más palpables en los paisajes intervenidos por el hombre (Pastrana, 2017). La influencia de estos factores también se ve en el departamento del Meta, el cual presenta una disminución de la riqueza de especies debido a procesos de urbanización y al desarrollo de actividades económicas tales como agricultura y ganadería, la transformación de las sabanas naturales y la pérdida de bosques remanentes (Etter, et al. 2015; Arévalo Ariza, 2016).

El departamento del Meta está conformado por 29 municipios, siendo Villavicencio su capital. Villavicencio por estar ubicado en el piedemonte de la cordillera Oriental de los Andes, presenta ecosistemas estratégicos donde aloja especies de aves migratorias y endémicas, además de mamíferos, reptiles, anfibios y alrededor de 400 especies de plantas (Resolución 2103 de 2012; Ortiz-Moreno, 2015). La conservación de la biodiversidad requiere la preservación de los hábitat específicos de la flora y fauna, por ello, para que esta conservación sea efectiva es necesario mantener las coberturas naturales (Ortiz-Moreno, 2015). A su vez, la cobertura vegetal mantiene la calidad del suelo, la riqueza hídrica (Ortiz-Moreno, 2015) y las interacciones biológicas como lo son la polinización, dispersión de semillas y la estructura de las cadenas tróficas indispensables para mantener la integridad de los ecosistemas (Cuevas et al. 2015; Díaz-Valenzuela, 2016; Josari et al, 2018; Quesada-Acuña et al, 2018; y Sharapova, 2018).

Sumado a lo anterior el Convenio sobre la Diversidad Biológica (CDB) (1992) ratifica en sus tres objetivos la necesidad de la conservación de la biodiversidad y que esta debe ser de interés común para toda la humanidad, por ende la utilización de todos los recursos naturales se debe hacer de tal manera que satisfaga las necesidades de las generaciones presentes y las futuras, dando una gestión sostenible a los recursos. Hay intereses de muchas ONG, gobierno nacional, departamental y municipal, universidades, centros de investigaciones y otras entidades, de conservar, mitigar y realizar inventarios faunísticos y florísticos, además de reforestar. Sin embargo existe un contrasentido administrativo y algunos proyectos de desarrollo se realizan a expensas del deterioro de los ecosistemas, es así, como a diario se otorgan licencias de construcción en áreas que son zonas estratégicas para la provisión de servicios ecosistémicos o el hábitat de especies. 
En el periodo del presidente Santos 2010-2014 la estrategia de crecimiento económico incluyó potenciar la vivienda e infraestructura, por lo que se aspiraba construir 1 millón de nuevas viviendas, de las cuales el $70 \%$ son viviendas de interés social. La mayoría de viviendas construidas en este macro proyecto fueron realizadas mediante métodos convencionales y no sustentables (Monroy \& Mario, 2015).

Unido a lo anterior en la ciudad de Villavicencio la expansión urbana y crecimiento de la población están causando pérdidas importantes de cobertura vegetal (Ortiz-Moreno, 2015; Caicedo et al, 2018). Debido a que el proceso de ocupación del territorio se ha presentado de forma desordenada y los planes de ordenamiento territorial diseñados como un instrumento técnico y normativo para ordenar el territorio urbano y rural no han sido eficaces a la hora de controlar las necesidades asociadas al crecimiento poblacional (Ortiz-Moreno \& Rodrigues-Pires, 2014), lo cual ha desencadenado una serie de consecuencias ambientales, económicas y sociales negativas para el desarrollo sostenible de la ciudad (OchoaAmaya, 2017).

En Villavicencio para cubrir las necesidades de vivienda, se desarrolló el proyecto de vivienda de interés social La Madrid, el cuál consta más de 1 . 000 soluciones de vivienda en la periferia del perímetro urbano al año 2000, desarrollando intervenciones urbanísticas sobre parte de las rondas hídricas del Caño Zuria y el humedal homónimo. Por todo lo anterior la presente investigación pretende describir la diversidad florística remanente en las rondas hídricas del Caño Zuria y el Humedal ubicados en el barrio La Madrid, ya que esta ha sido afectada por el conflicto entre vivienda de interés social y conservación de la biodiversidad en Villavicencio.

\section{DESCRIPCIÓN DEL ÁREA DE ESTUDIO Y METODOLOGÍA}

\section{Descripción del área de estudio}

El barrio La Madrid se encuentra ubicado en el suroriente del municipio de Villavicencio aledaño a los barrios Ciudad Porfía y Villa Juliana. Consta de soluciones de vivienda de interés social unifamiliares y multifamiliares (VILLAVIVIENDA, 2010). Comprende un área aproximada de 3.7ha de las cuales aproximadamente 2 ha corresponden a la zona de amortiguación del humedal La Madrid, a una altura de 453 msnm. Este barrio se creó mediante la resolución 591 del 26 de marzo de 2014 emitida por el Fondo Nacional de Vivienda (FONVIVIENDA) donde el Ministerio de Vivienda estableció la entrega de 1.122 casas a personas en condición de desplazamiento o vulnerabilidad registradas en el Departamento para la Prosperidad Social -DPS-, Red Unidos, y el Sistema de Identificación de Potenciales Beneficiarios de Programas Sociales (SISBEN) (Bueno, 2015) (Figura 1). 

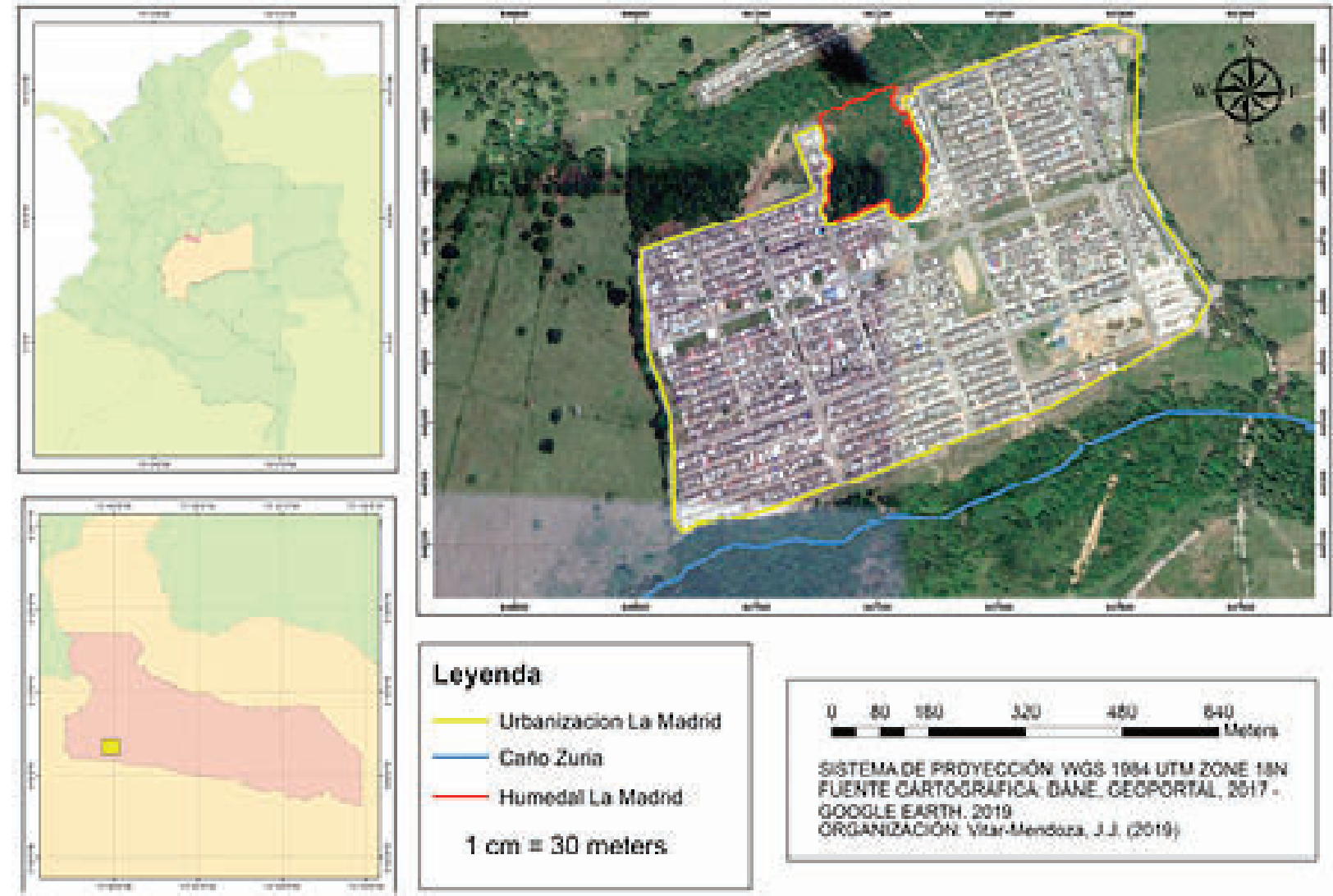

Figura 1. Ubicación del barrio La Madrid, Villavicencio.

Elaboró: Vitar-Mendoza (2019)

\section{METODOLOGÍA}

\section{Revisión bibliográfica}

Se realizó una búsqueda bibliográfica con temas relacionados a expansión urbana, vivienda de interés social y conflictos con la diversidad florística con los recursos disponibles en internet en portales oficiales, artículos e información suministrada por la actual Secretaria de Planeación del municipio de Villavicencio, Villavivienda y FONVIVIENDA.

\section{Muestreo en campo}

Se llevaron a cabo salidas de campo al barrio La Madrid (ciudad de Villavicencio) donde se colectaron muestras vegetales en estado reproductivo asociadas a las rondas hídricas del Caño Zuria y al humedal La Madrid, mediante un muestreo oportunista. Se hicieron las anotaciones en libreta de campo de los caracteres importantes para su posterior determinación; el material fue procesado empleando las técnicas de recolección de material vegetal propuesto por Villarreal, et al, (2004).

El material colectado fue determinado mediante la consulta de literatura especializada como: la Flora de la Guayana Venezolana (Pipoly et al, 1998), el Catálogo de plantas y líquenes de Colombia (Bernal et al, 2016). Adicionalmente, se consultaron los herbarios virtuales del Herbario Nacional Colombiano (COL), The U.S. National Herbarium (US), The New York Botanical Garden, The Missouri Botanical Garden (MO) y herbario LLANOS, 
además se contó con la ayuda de botánicas especialistas en las familias Melastomataceae y Poaceae. Para corroborar las sinonimias y los nombres aceptados se utilizó las bases disponibles en internet, como: Trópicos (http:// www.tropicos.org.) y Plant list (http:// www.theplantlist.org); también se revisaron trabajos de investigación donde se colecto material vegetal de la zona de estudio.

\section{RESULTADOS Y DISCUSIÓN}

\section{Vivienda de interés social y diversidad florística}

Las ciudades representan para muchos individuos y familias soluciones productivas y mejores condiciones de vida, sin embargo la forma del crecimiento urbano puede generar bienestar o perjuicios a los habitantes, debido a las condiciones de eficiencia en aprovechamiento del suelo, provisión de servicios, transporte, espacios públicos e interacción comunitaria (Ochoa-Amaya, 2017). Otro punto también importante es la biodiversidad presente en las zonas urbanas, aunque la mayoría de ecólogos consideran innecesario hacer estudios florísticos en esta áreas por el grado de perturbación (Kühn \& Klotz, 2006); hay otros ecólogos que si lo consideran importante, dado que, las ciudades pueden actuar como puentes de comunicación de un hábitat a otro para especies de alto valor de conservación, o pueden actuar como puntos de dispersión de especies invasoras que se establecen en zonas perturbadas. A su vez, una ciudad sostenible puede ser considerada como aquella que cuenta con zonas verdes saludables que sustentan una variedad de especies de plantas y animales en los jardines de las viviendas, en los vacíos urbanos y en el espacio público, lo cual contribuye positivamente al bienestar del hombre (Muratet et al, 2008).
Por otro lado, el departamento del Meta históricamente ha sufrido el flagelo del conflicto armado (Ortiz-Moreno, 2015). Según estadísticas, en los Llanos Orientales se registran 332.632 víctimas del conflicto armado (fuente: RNI, Red nacional de información, fecha de corte 01 de noviembre de 2019) cifra que se ve reflejada en la significativa migración de habitantes de los municipios del departamento, que han abandonado sus fincas y radicado en la ciudad de Villavicencio; a raíz de ello, la población en la capital ha crecido y los últimos gobiernos han tenido que diseñar planes de vivienda de interés social para cobijar a este grupo de personas, como ocurre en el barrio La Madrid, donde la mayoría de los habitantes son familias desplazadas.

El crecimiento demográfico es uno de los factores que acelera la perdida de la biodiversidad (Ortega et al, 2018), ya que se necesita espacio para el desarrollo urbanístico y ubicar a las poblaciones, ofreciéndoles una vivienda digna. Por tanto, las ciudades tienen que expandirse y crecer en su perímetro urbano y a raíz de ello, predios que antes eran considerados rurales, son absorbidos por la ciudad, por lo que entran a hacer parte de la zona urbana como lo que ocurre en el barrio La Madrid. Como el barrio está en construcción, no cuenta con los servicios básicos como puesto de salud, colegio, mobiliario urbano, áreas verdes apropiadas para los requerimientos de todas las franjas etarias de la población, entre otros.

El desarrollo urbanístico del barrio La Madrid se ha expandido en los dos ecosistemas analizados, los cuales presentan un alto grado de perturbación antrópica, siendo la situación del humedal más crítica, dado que no está protegido por el sistema municipal de áreas protegidas y no cuenta con una zona de amortiguación, ya que está ha sido absorbida por el barrio. 
La situación del bosque de galería de la ronda hídrica del Caño Zuria es también preocupante, lo cual es reconocido por CORMACARENA (Corporación Ambiental Regional con jurisdicción en el departamento del Meta) en el documento "Concepto técnico PM-GA-3.44.15.1696 de julio 29 de 2015". Donde se registra que la construcción del barrio está vertiendo las aguas lluvias al caño directamente y que la construcción ha deforestado gran parte de la ronda hídrica del caño Zuria, debido a lo anterior, se está generando el desplazamiento de la fauna como aves, herpetos y pequeños mamíferos por la falta de cobertura vegetal.

Como lo mencionan Ortiz-Moreno (2015), Buitrago et al, (2018) y Caicedo et al (2018), Villavicencio es considerado un corredor biológico entre las ecoregiones Andina y Orinoquía, además hace parte del cinturón verde del departamento del Meta por su ubicación geográfica, por lo que, la construcción de viviendas en lugares de importancia biológica como humedales y bosques de galería no debería autorizarse o en su defecto es necesario establecer lineamientos de construcción sostenible para edificaciones o viviendas de interés social, encaminados al mejoramiento de la calidad de vida de los habitantes y al ejercicio de actuaciones con responsabilidad ambiental y social (Decreto 1285 de 2015 titulo 7).
Según Pérez (2016) una vivienda de interés social, constituye uno de los ejes más importantes en la planificación urbana, ya que favorece la sustentabilidad y contribuye a elevar el bienestar reduciendo a la vez el impacto ambiental, sin embargo los modelos para la gestión de estos proyectos son subvalorados, dando preponderancia a la cantidad de viviendas y no al bienestar de sus habitantes y a la conservación ambiental.

En la figura 2 se presenta la diversidad de familias botánicas colectadas en las rondas hídricas del humedal La Madrid y el caño Zuria, se registraron 40 especies agrupadas en 23 familias; siendo Melastomataceae y Rubiaceae las familias mejor representadas en el área de estudio. En los ecosistemas las plantas son un grupo importante ya que mediante los frutos, semillas y néctar mantienen a la fauna que depende para su alimentación y estos a su vez son dispersores o polinizadores de las plantas (Aguirre \& Dirzo, 2008; Dàttilo et al, 2015), además las plantas les proveen refugio. Los frutos también son ampliamente utilizados por varias etnias y grupos humanos, ya que hacen parte de su dieta alimenticia (Núñez, et al, 2015) y obtienen de ellos fibras, tintas, medicina y utensilios de herramientas (Cruz, et al, 2017), entre otros. 


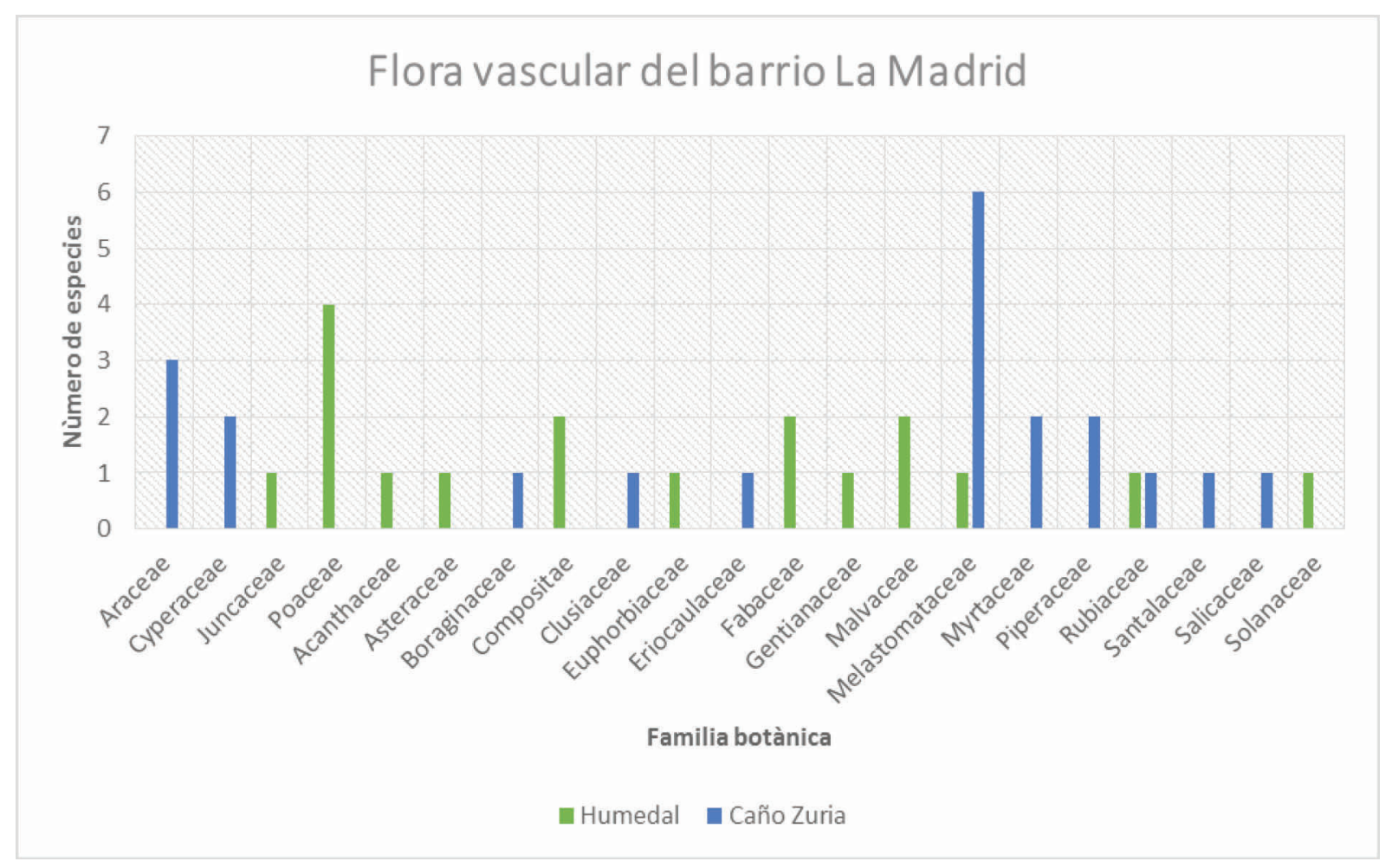

Figura 2. Comparación de la riqueza en familias botánicas del Humedal y Caño Zuria del barrio la Madrid

Fuente: autores.

\section{Flora vascular asociada al Humedal La Madrid}

En la flora asociada al humedal La Madrid se lograron identificar 19 especies agrupadas en 13 familias, siendo la familia Poaceae principalmente representada con cuatro (4) especies (anexo Tabla 1). Según la consulta de los libros rojos de Colombia Calderón et al, (2005) y Cárdenas \& Salinas (2007) hay dos especies que están en la categoría de amenaza de extinción preocupación menor (LC), las cuales son Andropogon bicornis L. y Solanum jamaicense Mill. Aunque el Catálogo de plantas y líquenes de Colombia (Bernal et al, 2016) las reportan con una amplia distribución en las regiones biogeográficas de Colombia. El género Juncus según Bernal et al, (2016) posee una distribución altitudinal desde los 900 a los 4.250 msnm, en esta investigación fue colectado a los $453 \mathrm{~m}$, por lo que se ampliaría el rango de distribución para este género.

A pesar de que el humedal La Madrid está siendo altamente afectado por acciones de deforestación (asociado a canalización para drenaje del terreno), descarga de escombros, extracción irregular de agua y el pastoreo no controlado por animales domésticos, posee una biodiversidad vegetal de gran importancia para la fauna local. Es por esta problemática que surge la necesidad de realizar este análisis de la flora, dado que el humedal ha sido sobreexplotado. Así mismo en Villavicencio los humedales son escasamente monitoreados por la administración municipal (Suazo \& Silva, 2014) y el hecho de que el humedal La Madrid no esté dentro del Sistema Nacional o Regional de Áreas Protegidas, ni protegido por 
la Corporación para Desarrollo Sostenible del Área de Manejo Especial la Macarena CORMACARENA, aumenta su vulnerabilidad ante las acciones antrópicas.

Hay que mencionar que un humedal es importante por su alto valor, escénico, cultural, biológico y su aporte de agua potable o regulación del ciclo hidrológico local. Adicionalmente estos ecosistemas proveen alimento y refugio a aves, primates y peces de gran importancia ecosistémica (Bastidas \& Sánchez, 2015; Franco-Montenegro et al, 2015.). Dado que el agua es el principal recurso para sostener la vida en la tierra, es importante hacer esfuerzos para recuperar y restaurar los humedales, especialmente en entornos urbanos. Así mismo el uso, protección y conservación de la diversidad biológica se han visto afectados por las acciones incorrectas de manejo de los recursos naturales. En este sentido, la flora recibe el impacto de la actividad antrópica y los cambios climáticos globales (Figueredo \& Acosta, 2008). Cabe resaltar que en el presente estudio se encontró dos especies que están en categoría de preocupación menor (LC), pero si se continua con la sobre explotación del humedal La Madrid pueden desaparecer localmente.

\section{Flora vascular asociada al caño Zuria}

Se colectaron 21 especies agrupadas en 11 familias (anexo Tabla 2), de estas la que presentó mayor número de especies colectadas fue Melastomaceae con seis especies, seguida por Araceae con tres especies; sin embargo CORMACARENA (2014) reporta para el humedal Zuria, el cual es influenciado por el caño del mismo nombre, 41 especies agrupadas en 34 familias botánicas, esto puede estar relacionado con el esfuerzo de muestreo.

Por otro lado de las especies encontradas en el presente estudio, Psychotria poeppigiana Mûll., está dentro de la categoría de amenaza de extinción preocupación menor (LC) (Calderón et al, 2005; Cárdenas \& Salinas, 2007), ya que en el Catálogo de plantas y líquenes de Colombia (Bernal et al, 2016) la reportan con una amplia distribución en el país. Adicionalmente, el presente trabajo genera importantes aportes tales como la ampliación de la distribución geográfica de Miconia dodecandra (Desr) Cogn., a partir de los $444 \mathrm{~m}$, dado que Bernal et al, (2016) la reporta desde los 775 msnm.

A pesar de su importancia biológica la ronda hídrica del caño Zuria está presentando una alta tasa de deforestación, implementación de cultivos y pastos para ganadería extensiva en su entorno local (CORMACARENA, 2014), por lo que el caudal del caño ha disminuido.

EI IDEAM (2008) define a la vegetación arbórea que crece en las márgenes de los cursos de agua, ya sean permanentes o temporales como bosques de galería, por lo que este ecosistema es una comunidad vegetal compleja y frágil, que cumple un papel fundamental en términos ecológicos, hidrológicos y de biodiversidad para la conservación de las fuentes hídricas (Meli et al, 2017). La disposición vertical de la vegetación arbórea del bosque de galería es primordial para mantener la calidad del agua, amortiguar los procesos de sedimentación de los lechos de los ríos (Granados et al, 2006), proporcionar protección contra la erosión del suelo y proveer un hábitat para organismos acuáticos y terrestres (Camacho et al, 2006).

El caño Zuria es afluente del río Negro dentro de la cuenca del Guayuriba que finalmente desemboca en el rio Meta y este a su vez, en el río Orinoco. Sumado a lo anterior la comunidad que habita el barrio La Madrid usa el agua para consumo y recreación; por lo que es importante restaurar y recuperar los servicios ecosistémicos de este caño y darle un uso sostenible. 


\section{CONCLUSIONES}

La flora asociada al humedal La Madrid está compuesta por 19 especies agrupadas en 13 familias, siendo la familia Poaceae la mejor representada con cuatro (4) especies. Adicionalmente en este humedal hay dos especies que están en la categoría de amenaza de extinción preocupación menor (LC), las cuales son A. bicornis y S. jamaicense, además se amplía el rango de distribución para Colombia del genero Juncus.

En la flora asociada al bosque de galería de la ronda hídrica del caño Zuria se colectaron 21 especies agrupadas en 11 familias, de estas la que presento mayor número de especies colectadas fue Melastomaceae con seis especies, seguida por Araceae con tres especies. De las especies identificadas, según los libros rojos $P$. poeppigiana está dentro de la categoría de amenaza de extinción preocupación menor (LC), en tanto que el presente estudio amplía la distribución de $M$. dodecandra a partir de los $444 \mathrm{msnm}$.

En el humedal La Madrid y la ronda hídrica del caño Zuria adyacentes al barrio La Madrid, se registraron 40 especies agrupadas en 23 familias; siendo Melastomataceae y Rubiaceae las familias mayormente representadas. En los análisis realizados fue evidente que el desarrollo urbano no sostenible afecta la biodiversidad de la flora y la oferta de servicios ecosistémicos asociada.

\section{LITERATURA CITADA}

Aguirre, A., \& Dirzo, R. (2008). Effects of fragmentation on pollinator abundance and fruit set of an abundant understory palm in a Mexican tropical forest. Biological Conservation, 141(2), 375-384.

Arévalo Ariza, Y. D. P. (2016). Descripción cualitativa y aproximación bibliográfica del impacto antrópico generado a las preferencias de microhábitat de un ensamblaje de anuros en tres hábitats del jardín botánico de Villavicencio (Meta), durante el periodo de lluvias (Bachelor's thesis, Universidad Militar Nueva Granada).

Bastidas, J. A. O., \& Sánchez, M. T. C. (2015). El análisis de correspondencias y la valoración social de la flora del humedal el Coroncoro de Villavicencio. Pag. 445-446. En Contreras, J.M.; Batanero, C.; Godino, J.; Cañadas, G.; Arteaga, P.; Molina, G.; Gea, M. y López, M. (Eds.), Didáctica de la Estadística, Probabilidad y Combinatoria, (2). Granada.

Bernal, R., Gradstein, S. \& Celis, M. (2016). Catálogo de plantas y líquenes de Colombia. Instituto de Ciencias Naturales, Universidad Nacional de Colombia, Bogotá. WWW. catalogoplantascolombia.unal.edu.co.http://catalogoplantascolombia.unal.edu.co.

Bueno, C. H. (17 de Diciembre de 2015). region365 periodismo alternativo. Obtenido de http://www. region365.com/

Buitrago, D., Ceballos, L., Ortiz-Moreno, M.L. (2018). Estrategia para la conservación de primates diurnos en el área urbana de Villavicencio con base a su distribución y conflictos. Tesis de pregrado, programa de Biología, Universidad de los Llanos. Pp. 130.

Caicedo, R. I. V., Guarín, K. J. D., \& Perdomo, Y. R. (2018). Composición y diversidad del banco de semillas en áreas urbanas fragmentadas de piedemonte, Villavicencio, Colombia. Ingenierías USBMed, 9(1), 86-96.

Camacho, R. F., I. Trejo y C. Bonfil. (2006). Estructura y composición de la vegetación ribereña de la Barranca del río Tembembe, Morelos, México. Boletín de la Sociedad Botánica de México 78: 17-31.

Cárdenas, L. D. \& N.R. Salinas (Eds). (2007). Libro rojo de plantas de Colombia. Volumen 4. Especies maderables amenazadas. Primera parte. Serie libros rojos de especies amenazadas Ministerio de Ambiente, Vivienda y Desarrollo Territorial. $232 \mathrm{Pp}$.

Cuevas, E., Álvarez, A., y de los Ángeles Pérez, M. (2015). Efecto de la fragmentación del hábitat en el éxito reproductivo de Fuchsia parviflora, una especie dioica. Biológicas Revista de la DES Ciencias Biológico Agropecuarias Universidad Michoacana de San Nicolás de Hidalgo, 16(1), 30-32.

Cruz-Fernández, J., Suarez-Suarez, L. S., OrtizMoreno, M.L. (2017). Catalogo comentado y análisis espacial del genero Clusia (Clusiaceae) 
en el departamento del Meta. Tesis de pregrado, programa de Biología, Universidad de los Llanos. Pp 114.

Dàttilo, W., Aguirre, A., Quesada, M., \& Dirzo, R. (2015). Tropical forest fragmentation affects floral visitors but not the structure of individualbased palm-pollinator networks. PloS one, 10(3).

Díaz-Valenzuela, R. (2016). Historia natural, ecología y análisis de la interacción planta-colibrí en un paisaje mexicano, bajo dos aproximaciones teóricas: escalamiento en ecología y redes de interacciones complejas. Tesis doctorales. Facultad de Ciencias Ambientales y Recursos Naturales, Universidad de Alicante. Alicante.

Etter, A., Andrade, A., Amaya, P., \& Arévalo, P. (2015). Estado de los ecosistemas colombianos-2014: una aplicación de la metodología de lista roja de ecosistemas. Bogota, Colombia: From Alaska to Patagonia: IUCN Red List of the Continental Ecosystems of the Americans. Recuperado de http://iucnrle.org/static/media/ uploads/references/published-assessments/ etter-etal-2015-national-rle-assessment-finalreport-colombia-sp.pdf. Google Scholar.

Franco-Montenegro, A., Torres-Pérez, R. E., \& Obando-Bastidas, J. A. (2015). Valoración social del recurso natural fauna en el humedal Coroncoro de Villavicencio. Producción + Limpia 10 (2).

Figueredo Cardona, L. M., \& Acosta Cantillo, F. (2008). Objetos de conservación de la flora y la vegetación de los cerros calizos costeros de la reserva de la biosfera Baconao, Santiago de Cuba. Foresta Veracruzana, 10(2).

Giraldo-cañas, D. (2001). Análisis florístico y fitogeográfico de un bosque secundario pluvial andino, Cordillera Central (Antioquia, Colombia). Darwiniana, 39: 187-199

Granados S., D., M. A. Hernández y G. F. López R. (2006). Ecología de las zonas ribereñas. Revista Chapingo Serie Ciencias Forestales y del Ambiente 12 (1): 55-69.

IDEAM, IGAC y Cormagdalena. (2008). Mapa de Coberturas de la Tierra, cuenca Magdalena - Cauca, metodología Corine Land Cover adaptada para Colombia a escala 1:100.000. Instituto de $\mathrm{Hi}-$ drología, Meteorología y Estudios Ambientales (Ideam), Instituto Geográfico Agustín Codazzi (IGAC), Corporación Autónoma Regional del Río Grande de la Magdalena (Cormagdalena). Bogotá D.C., Colombia. 200 pp
Josari, G., \& Morante Dávila, L. R. (2018). Diagnóstico de los recursos territoriales turísticos para el diseño de una ruta en el distrito de San Felipe, Jaen-Cajamarca. Facultad de Ciencias empresariales. Escuela académica profesional de turismo y negocios, universidad Señor de Sipan. Perú.

Kühn, I. \& Klotz, S. (2006). Urbanization and homogenization comparing the floras of urban and rural areas in Germany. Biological Conservation 127: 292-300.

Meli, P., L. Ruiz, R. Aguilar, A. Rabasa, J. M. Rey B. y J. Carabias. (2017). Bosques ribereños del trópico húmedo de México: un caso de estudio y aspectos críticos para una restauración exitosa. Madera y Bosques 23 (1): 181-193

Minorta, V. \& Rangel, O., (2014). Amenazas sobre la flora, la vegetación y los ecosistemas de la Orinoquia Colombiana, Pag. 785-806. En: RangelCh. O. 2014. Colombia diversidad biótica XIV, la región de la Orinoquia de Colombia. Universidad Nacional de Colombia. Bogotá.

Monroy, S., \& Mario, J. (2015). Construcción sostenible, una alternativa para la edificación de viviendas de interés social y prioritario. (Bachelor's thesis), Facultad de Ingeniería. Universidad Católica de Colombia.

Muratet, A., Porcher, E., Devictor, V., Arnal, G., Moret, J., Wright, S., \& Machon, N. (2008). Evaluation of floristic diversity in urban areas as a basis for habitat management. Applied vegetation science, 11(4), 451-460.

Myers, N., Mittermeier, R.A., da Fonseca, G.A.B. \& Kent, J. (2000). Biodiversity hotspots for conservation priorities. Nature, 403: 853-858.

Núñez, L. A., Isaza, C., \& Galeano, G. (2015). Ecología de la polinización de tres especies de Oenocarpus (Arecaceae) simpátricas en la Amazonia Colombiana. Revista de Biología Tropical, 63(1).

Ochoa-Amaya, J. M. (2017). Uso y tenencia de la tierra. Factores históricos y planes de ordenamiento territorial-veredas Barcelona, Cocuy y Zuría-Villavicencio, Colombia. Direito da Cidade, 9(4).

Ortega, D. J. P., Solarte, J. G., Díaz, J. J. P., \& Pompêo, M. L. M. (2018). Dinámica Espacio-Temporal Del Uso Del Suelo Y Su Efecto Sobre La Degradación De Los Recursos Hídricos: Caso De La Cuenca Del Río Juqueri-Brasil. Revista de Investigación Agraria y Ambiental, 9(1). 
Ortiz-Moreno, M. L., \& Rodrigues-Pires, J. S. (2014). Aplicación de la legislación ambiental y territorial en municipios capitales: Estudio de caso Villavicencio (Colombia). Orinoquia, 18(2).

Ortiz-Moreno, M.L., (2015). Analise Interacäo entre ordenamento territorial e biodiversidade: estudo de caso em Villavicencio, (Meta - Colombia). Universidad Federal de Säo Carlos. Säo Carlos, Brasil

Pastrana, J. (2017). Análisis del impacto ambiental en la microcuenca de la quebrada La Perdiz asociada a la zona urbana del municipio de Florencia-Caquetá. Tesis de pregrado. Facultad de Ciencias Económicas, Contables y Administrativas, Universidad de Manizales.

Pérez, A. L. P. (2016). El diseño de la vivienda de interés social: la satisfacción de las necesidades y expectativas del usuario. Revista de Arquitectura, (18), 67-75.

Pipoly, J., Kearns, D., Berry, P., (1998). Clusia. Pag. 260-294. En Steyermark, J., Berry, P., Holst, B., (1998). Flora of the Venezuelan Guyana, Caesalpiniaceae-Ericaceae. Missouri Botanical Garden. Vol. 4. Missouri, USA.

Quesada-Acuña, S. G., Martínez, C. P., Alán, O. R., \& Gastezzi-Arias, P. (2018). Dispersión de semillas por aves residentes en bosque ribereño urbano del río Torres, San José, Costa Rica. UNED Research Journal, 10(1), 48-56.

Rangel-Ch, J. O. (2015). La biodiversidad de Colombia: significado y distribución regional. Revista de la Academia Colombiana de Ciencias Exactas, Físicas y Naturales, 39(151), 176-200.

Sierra-Giraldo, J. A., Sanin, D., Coca, L.F. \& PosadaHerrera, J.M. (2013). Araceae in a High Andean Forest of the Colombian Occidental Cordillera (Natural National Park Tatamá). Aroideana., 36: 76-91.

Sharapova, A. (2018). Patrones en la diversidad de especies, abundancia y grado de generalismo de los polinizadores en diferentes ambientes en la isla de Mallorca. Facultad de Ciencias. Universidad de les Illes Balears.
Suazo, G. G., \& Silva, L. T. (2014). Importancia ecosistémica del humedal El Coroncoro sobre el barrio Manantial en la ciudad de Villavicencio/Meta. Boletín Semillas Ambientales, 8(2).

Uvidia, H., Ramírez, J. L., Leonard, I., Vargas, J. C., Verdecia, D., \& Andino, M. (2015). Inventario de la sucesión vegetal secundaria en la provincia Pastaza, Ecuador. REDVET. Revista Electrónica de Veterinaria, 16(11).

Villarreal, H., Álvarez, M., Córdoba, S., Escobar, F., Fagua, G., Gast, F., Mendoza, H., Ospina, M., Umaña, A.M., (2004). Manual de métodos para el desarrollo de inventarios de biodiversidad. Programa de Inventarios de Biodiversidad. Instituto de Investigación de Recursos Biológicos Alexander von Humboldt. Bogotá, Colombia, 236.

\section{AGRADECIMIENTOS}

Los autores agradecemos a la comunidad del barrio La Madrid y en especial al señor Oscar Soto, quien colaboró en las actividades de muestreo y a la Directora de la Fundación WiIliam Barrios (Organización No Gubernamental) Nathaly Rojas por su acompañamiento y suministro de información. Al igual agradecemos a los estudiantes del programa de Ingeniería Agronómica Juan Daniel Larrota Escobar y Roney Fabián Alarcón López por su colaboración en la colecta y procesamiento del material vegetal, además al biólogo Juan Jair Vitar-Mendoza quién colaboró en la elaboración de cartografía temática del área de estudio. Agradecemos también a las profesoras Luz Mila Quiñones y Mónica Medina del área de botánica de la Facultad de Ciencias Básicas e Ingenierías de la Universidad de los Llanos, por su ayuda para la identificación de algunas de las especies colectadas.
Conflicto de Intereses Los autores declaran no tener ningún conflicto de intereses

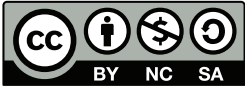

